\title{
L'HOSPITAL-TYPE RULES FOR MONOTONICITY: DISCRETE CASE
}

\author{
IOSIF PINELIS
}

Abstract. Let $f:=\left(f_{n}\right)$ and $g:=\left(g_{n}\right)$ be two sequences, and let $r:=\frac{f}{g}$ and $\rho:=\frac{\delta f}{\delta g}$, where $(\delta f)_{n}:=\delta f_{n}:=f_{n}-f_{n-1}$. In particular, it is shown that, if $r$ is monotonic, then the monotonicity pattern of $\rho$ may switch at most once (from increase to decrease or vice versa). "Continuous" versions of such results were obtained earlier by the author.

Mathematics subject classification (2000): 26A48, 11B99, 39A70.

Key words and phrases: 1'Hospital-type rules for monotonicity, sequences.

\section{REFERENCES}

[1] G. D. ANDERSON, S.-L. Qiu, M. K. VAmANAMURTHY, AND M. Vuorinen, Generalized elliptic integrals and modular equations, Pacific J. Math. 192 (2000) 1-37.

[2] G. D. Anderson, M. K. VAmanamurthy, AND M. Vuorinen, Inequalities for quasiconformal mappings in space, Pacific J. Math. 160 (1993) 1-18.

[3] G. D. Anderson, M. K. Vamanamurthy, And M. Vuorinen, Conformal Invariants, Inequalities, and Quasiconformal Maps, Wiley, New York, 1997.

[4] G. D. Anderson, M. K. Vamanamurthy, AND M. Vuorinen, Topics in special functions, in Papers on Analysis, Rep. Univ. Jyväskylä Dep. Math. Stat., no. 83 (2001), Jyväskylä, Finland, pp. 5-26.

[5] G. D. Anderson, M. K. Vamanamurthy, And M. Vuorinen, Monotonicity of some functions in calculus, Amer. Math. Monthly, 113 (2006), 805-816.

[6] R. E. Barlow, A. W. Marshall, and F. Proshan, Properties of probability distributions with monotone hazard rate, Ann. Math. Statist. 34 (1963) 375-389.

[7] R. P. BOAS, JR., Lhospital's Rule Without Mean-Value Theorems, Amer. Math. Monthly 769 (1969) 1051-1053.

[8] I. CHAVEL, Riemannian Geometry-A Modern Introduction, Cambridge University Press, Cambridge, 1993.

[9] J. CHEEGER, M. GROMOV, AND M. TAYLOR, Finite propagation speed, kernel estimates for functions of the Laplace operator, and the geometry of complete Riemannian manifolds, J. Diff. Geom. 17 (1982) $15-54$.

[10] M. GROMOV, Isoperimetric inequalities in Riemannian manifolds, in Asymptotic Theory of Finite Dimensional Spaces, Lecture Notes in Math., no. 1200, Springer-Verlag, Berlin, 1986, pp. 114-129.

[11] R. L. HALL, M. KANTER, AND M. D. PERLMAN, Inequalities for probability content of a rotated square and related convolutions, Ann. Statist. 8 (1980) 802-813.

[12] I. PINELIS, Extremal probabilistic problems and Hotelling's $T^{2}$ test under symmetry condition, (preprint, 1991, http: / / arXiv: math / $0701806 \mathrm{v} 1$ ); a shorter version of the preprint appeared in Ann. Statist. 22 (1994) 357-368.

[13] I. PINELIS, Fractional sums and integrals of $r$-concave tails and applications to comparison probability inequalities. Advances in stochastic inequalities, (Atlanta, GA, 1997), 149-168, Contemp. Math. 234 Amer. Math. Soc., Providence, RI, 1999.

[14] I. PINELIS, L'Hospital type rules for monotonicity, with applications, J. Inequal. Pure Appl. Math. 3 (2002), article 5, 5 pp. (electronic).

[15] I. PINELIS, L'Hospital type rules for oscillation, with applications, J. Inequal. Pure Appl. Math. 2 (2001), article 33, 24 pp. (electronic). 
[16] I. PINELIS, Monotonicity properties of the relative error of a Padé approximation for Mills' ratio, J. Inequal. Pure Appl. Math. 3 (2002), article 20, 8 pp. (electronic).

[17] I. PINELIS, L'Hospital type rules for monotonicity: Applications to probability inequalities for sums of bounded random variables, J. Inequal. Pure Appl. Math. 3 (2002), article 7, 9 pp. (electronic).

[18] I. PINELIS, L'Hospital rules for monotonicity and the Wilker-Anglesio inequality, Amer. Math. Monthly 111 (2004) 905-909.

[19] I. PINELIS, L'Hospital-Type Rules for Monotonicity, and the Lambert and Saccheri Quadrilaterals in Hyperbolic Geometry, J. Inequal. Pure Appl. Math. 64 (2005), Article 99, 12 pp. (electronic).

[20] I. PINELIS, On L'Hospital-Type Rules for Monotonicity, J. Inequal. Pure Appl. Math. 72 (2006), Article 40, 19 pp. (electronic).

[21] I. PINELIS, Binomial upper bounds on generalized moments and tail probabilities of (super)martingales with differences bounded from above, IMS Lecture Notes-Monograph Series, High Dimensional Probability, Vol. 51 (2006). Institute of Mathematical Statistics, 2006. DOI: 10.1214/074921706000000743. http: //arxiv.org/abs/math.PR/0512301.

[22] I. PINELIS, Normal domination of (super)martingales, (2006) Electronic J. Probab., Vol. 11, Paper 39, 1049-1070, http://www. math.washington.edu/ ejpecp/viewarticle.php?id $=1648 \&$ layout $=$ abstract .

[23] I. PINELIS, On inequalities for sums of bounded random variables, (2006) Preprint, http:// arxiv.org/abs/math.PR/0603030.

[24] I. PINELIS, Toward the best constant factor for the Rademacher-Gaussian tail comparison, (2006) Preprint, http://arxiv.org/abs/math.PR/0605340. To appear in ESAIM Probability and Statistics

[25] I. PINELIS, "Non-strict" l'Hospital-type rules for monotonicity: intervals of constancy, J. Inequal. Pure Appl. Math. 81 (2007), Article 14, 8 pp. (electronic). 